\title{
PAHS AND THE ISM IN METAL-POOR STARBURSTS
}

\author{
L.K. Hunt ${ }^{1}$, Y.I. Izotov ${ }^{2}$, M. Sauvage ${ }^{3}$ and T.X. Thuan ${ }^{4}$
}

\begin{abstract}
We characterize PAH populations in 22 metal-poor blue compact dwarf galaxies (BCDs), 16 of which have an oxygen abundance $12+\log (\mathrm{O} / \mathrm{H}) \lesssim 8$. This is the largest sample ever studied at such low metallicities. The relative PAH intensities of the $6.2,7.7,8.6$ and $11.3 \mu \mathrm{m}$ features in these BCDs suggest a deficit of small PAH carriers, or alternatively, an excess of large ones at these low abundances.
\end{abstract}

\section{Introduction}

PAH emission dominates the mid-infrared spectra of star-forming galaxies (Brandl et al. 2006; Smith et al. 2007), and contributes importantly to their IR energy budget. In most star-forming galaxies, PAH emission has a surprisingly narrow range of properties, well characterized by a "standard set" of features (Smith et al. 2007). However, first ISO and later Spitzer have shown that metal-poor starforming galaxies are deficient in PAH emission (Madden et al. 2006; Wu et al. 2006; Engelbracht et al. 2008), similar to the observed deficit in CO emission (e.g., Taylor et al. 1998). Neither PAH nor CO emission is generally detected below $12+\log (\mathrm{O} / \mathrm{H}) \sim 8$, consistent with the idea that PAHs and $\mathrm{CO}$ form in similar conditions within molecular clouds (Sandstrom et al. 2010, see Sandstrom et al. elsewhere in this volume).

Although it has generally been concluded that the reason for this PAH deficiency is low metallicity, it is not yet clear whether it is the metallicity directly, or rather the indirect effects of a low metallicity environment. Previous work with ISO (e.g., Galliano et al. 2005; Madden et al. 2006) examined this question, but the metallicity of the galaxies studied by ISO did not go below $12+\log (\mathrm{O} / \mathrm{H}) \sim 8.2$, making it difficult to probe the physical conditions where PAH emission is effectively suppressed. Spitzer/IRS added more observations of

\footnotetext{
1 INAF-Osservatorio di Arcetri, Firenze, Italy

2 Main Astronomical Observatory, Kiev, Ukraine

3 CEA, Saclay, France

4 University of Virginia, Charlottesville, USA
} 
the PAHs in low-metallicity dwarf galaxies, but up to now only five objects with $12+\log (\mathrm{O} / \mathrm{H}) \lesssim 8(\mathrm{Wu}$ et al. 2006$)$ were available for study.

Here, we present observations of PAH features in a new sample of 22 metalpoor blue compact dwarf galaxies (BCDs), 16 of which have $12+\log (\mathrm{O} / \mathrm{H}) \lesssim 8$; this more than triples the number of such dwarfs with PAH spectra. We examine in detail the PAH emission in our sample, and show for the first time that some PAHs can survive even in extremely metal-poor environments.

\section{Observations and analysis}

In the context of our GO Spitzer program (PID 3139), we obtained IRS spectra in the low- and high-resolution modules (SL, SH, LH: Houck et al. 2004). The sample definition and data reduction are described in detail by Hunt et al. (2010). In order to directly compare the results for our low-metallicity sample with those of more metal-rich galaxies, we analyzed our IRS spectra with PAHFIT (Smith et al. 2007), an IDL procedure which was developed for and applied to the Spitzer Nearby Galaxy Survey (SINGS: Kennicutt et al. 2003). PAHFIT is particularly suited for separating emission lines and PAH features (e.g., the PAH blend at $12.6-12.7 \mu \mathrm{m}$ and the [NeII] line at $12.8 \mu \mathrm{m})$, as well as measuring faint PAH features superimposed on a strong continuum. PAHFIT overcomes this problem by fitting simultaneously the spectral features, the underlying continuum, and the extinction. Drude profiles are fitted to the PAH features, and Gaussian profiles to the molecular hydrogen and fine-structure lines.

\section{Results}

The $7.7 \mu \mathrm{m}$ blend is the most common PAH feature in our sample, detected in 15 of 22 objects. The $7.7 \mu \mathrm{m}$ feature is also the strongest one, comprising roughly $49 \%$ of the total PAH power. The remaining PAHs are significantly weaker, and less frequently detected: 13 BCDs show the $11.2-11.3 \mu \mathrm{m}$ PAH, 9 the $8.6 \mu \mathrm{m}$ feature, and 7 the $6.2 \mu \mathrm{m}$ PAH. No $17 \mu \mathrm{m}$ PAH features were detected. The 6.2, 7.7, 8.6, 11.3 , and $12.6 \mu \mathrm{m}$ features by themselves constitute $\sim 72 \%$ of total $\mathrm{PAH}$ power in BCDs; the SINGS sample has about $\sim 85 \%$ of total PAH power in these bands (Smith et al. 2007). The remainder of the emission is in the weaker features.

\subsection{Fractional power of strongest features}

The fractional power of the four strongest features relative to the total PAH luminosity is illustrated in Figure 1. The horizontal lines in each panel correspond to the SINGS medians (dashed) and the BCD means (dotted). The BCD means are calculated taking into account all galaxies with $7.7 \mu \mathrm{m}$ detections; thus they are a sort of weighted average which considers frequency of detection together with intensity. This is why the mean for the $6.2 \mu \mathrm{m}$ PAH lies below most of the BCD data points: that feature was detected only in 7 galaxies, while the other features in Figure 1 were detected with a frequency more similar to the $7.7 \mu \mathrm{m}$ PAH. The relative PAH strengths for the BCD and SINGS 6.2 and $7.7 \mu \mathrm{m}$ features are virtually indistinguishable: 0.10 vs. 0.11 for $6.2 \mu \mathrm{m}$, and 0.49 vs. 0.42 for $7.7 \mu \mathrm{m}$ (Smith et al. 2007). However, for the longer wavelength PAHs at 8.6 


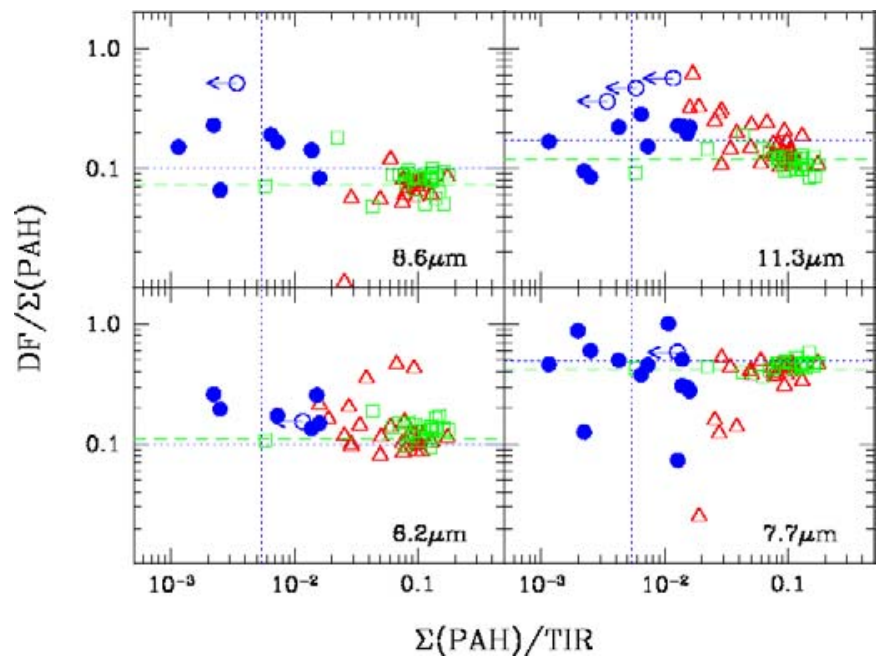

Fig. 1. PAH strengths relative to total PAH luminosity vs. total PAH power normalized to total IR luminosity (TIR). The four panels show the the following dust features (DF): top $8.6 \mu \mathrm{m}, 11.3 \mu \mathrm{m}$, and $6.2 \mu \mathrm{m}, 7.7 \mu \mathrm{m}$ features. BCDs are plotted as filled (blue) circles; open (green) squares correspond to SINGS HII nuclei; and open (red) triangles to SINGS AGN (Smith et al. 2007). The horizontal dashed lines give the SINGS sample medians (Smith et al. 2007), and the dotted ones the means for the BCD sample, taking into account all objects with $7.7 \mu \mathrm{m}$ detections. The vertical dotted line corresponds to the BCD mean PAH power normalized to TIR.

and $11.3 \mu \mathrm{m}$, the BCD fractions are $\gtrsim 40 \%$ larger: 0.10 vs. 0.07 (8.6) and 0.17 vs. 0.12 (11.3). Although with considerable scatter, the BCD mean PAH relative fractions for these features tend to exceed even the 10 to 90 th percentile spreads of the SINGS galaxies.

\subsection{Profile widths and central wavelengths}

Figure 2 shows the mean Drude profile widths and central wavelengths of six aromatic features detected at $\gtrsim 3 \sigma$ in our sample; the numbers of BCDs with each feature are given in parentheses. The analogous quantities for the SINGS galaxies are also plotted. In no case, were the PAHFIT profiles fixed to the SINGS galaxy parameters able to fit the BCD data; every BCD was better fitted by allowing the Drude profile widths (FWHMs) and central wavelengths to vary. In some cases, the differences between the $\chi_{\nu}^{2}$ with the SINGS "standard" profile parameters and the best-fit parameters were small, $\lesssim 15 \%$; but in others, the improvement of $\chi_{\nu}^{2}$ was almost a factor of 2 . The only systematic difference between the two samples is the narrower profile width for the $8.6 \mu \mathrm{m}$ feature; only the very broadest BCD profiles are as broad as those in more metal-rich systems. 


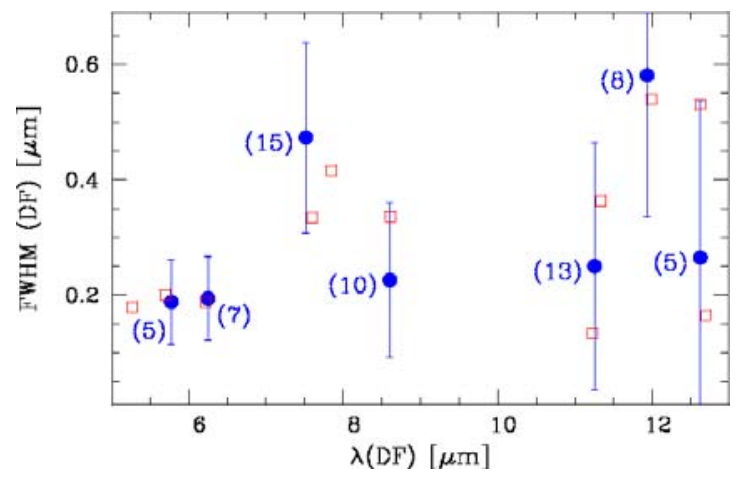

Fig. 2. Median Drude profile widths (FWHM) and central wavelengths for seven aromatic features detected $(\gtrsim 3 \sigma)$ in our sample. The error bars are the standard deviations of the measurements, and the numbers in parentheses are the BCDs with significant detections. Open squares correspond to SINGS sample means (Smith et al. 2007).

\subsection{Lack of small PAHs at low metallicity?}

The larger relative intensities of the 8.6 and $11.3 \mu \mathrm{m}$ feature in BCDs and the narrow profile width of the $8.6 \mu \mathrm{m}$ PAH could be due to a different size distribution of PAH populations at low metal abundances. Bauschlicher et al. (2008) find that the $8.6 \mu \mathrm{m}$ band arises from large PAHs, with $N_{\min } \gtrsim 100$ carbon atoms. Hence, they suggest that the relative intensity of the $8.6 \mu \mathrm{m}$ band can be taken as an indicator of the relative amounts of large and small PAHs in a given population. The large $8.6 \mu \mathrm{m}$ intensity relative to total $\mathrm{PAH}$ power in BCDs could be a signature of fewer small PAHs (or more larger ones) at low metallicity. The relative lack of small PAHs could also explain the low detection rate of the $6.2 \mu \mathrm{m}$ feature, since most of its intensity comes from PAHs with less than $100 \mathrm{C}$ atoms (Schutte et al. 1993; Hudgins et al. 2005). The size of a PAH molecule grows with increasing numbers of carbon atoms (Draine \& Li 2007). For a given maximum number of carbon atoms $N_{\max }$, a larger minimum number $N_{\min }$ theoretically gives narrower profiles (Joblin et al. 1995; Verstraete et al. 2001). Although fitting intrinsically asymmetric PAH profiles by symmetric Drude profiles in PAHFIT is a simplification, the narrower width of the $8.6 \mu \mathrm{m}$ feature could be a signature of relatively larger PAHs at low metallicity. The difference disappears at $7.6 \mu \mathrm{m}$ and is less pronounced at longer wavelengths than $8.6 \mu \mathrm{m}$ because both small and large PAH sizes contribute to these bands ${ }^{1}$ (Schutte et al. 1993; Bauschlicher et al. 2009).

Another indication that low-metallicity BCDs may be lacking the smallest size PAHs comes from a correlation analysis. In a detailed study of Galactic HiI regions, young stellar objects, reflection and planetary nebulae (RNe, PNe), and evolved stars, Hony et al. (2001) found only a few correlations among PAH features. One of these is between 6.2 and the $12.7 \mu \mathrm{m}$ emission, which is shown for our sample of BCDs in Figure 3; only galaxies with $\gtrsim 3 \sigma$ detections in at least two features are plotted. Most galaxies with $6.2 \mu \mathrm{m}$ PAH detections and $12+\log (\mathrm{O} / \mathrm{H}) \geq 8.1$ (filled circles) are similar to the HII regions studied by Hony et al. (2001). On the other

\footnotetext{
${ }^{1}$ The $7.8 \mu \mathrm{m}$ band may be dominated by larger PAHs only (Bauschlicher et al. 2009); but this band is present in only two of the BCDs in our sample, Haro 3 and II Zw 70.
} 


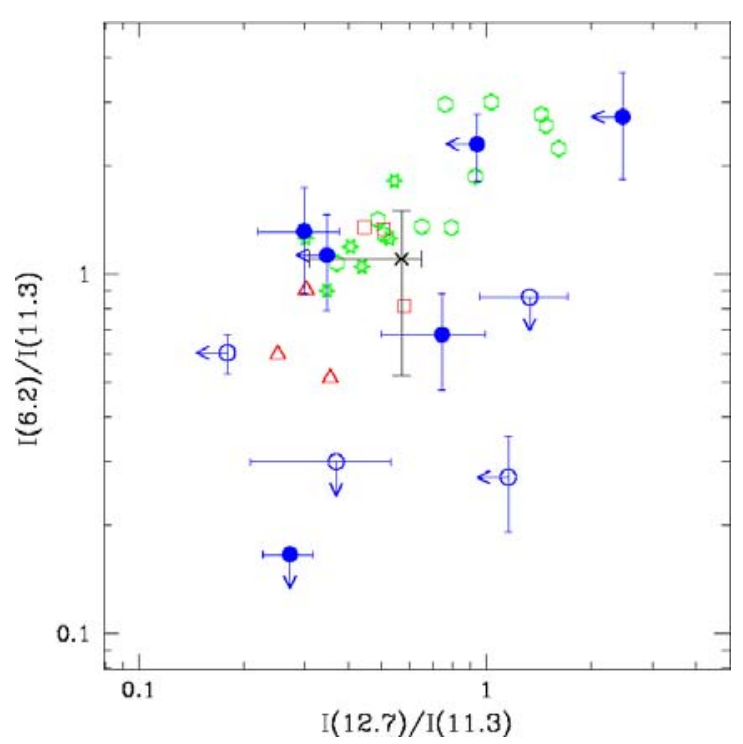

Fig. 3. The integrated flux in the $\mathrm{PAH}$ features at $6.2 \mu \mathrm{m}$, $11.3 \mu \mathrm{m}$, and $12.7 \mu \mathrm{m}$, plotted in two sets of ratios: $6.2 / 11.3$ vs. $12.7 / 11.3$. Only those BCDs with at least one ratio with $11.3 \mu \mathrm{m}$ are shown: filled (blue) circles correspond to those BCDs with $12+\log (\mathrm{O} / \mathrm{H}) \geq 8.1 ; \quad$ open ones to those objects with lower metallicities. The other data are taken from Hony et al. (2001) and correspond to PNe, RNe, intermediate-mass star-forming regions and HiI regions. The SINGS average is shown as a $\times$, with error bars reporting standard deviations over the sample (Smith et al. 2007).

hand, BCDs with lower $\mathrm{O} / \mathrm{H}$ occupy a region of the plot with small 6.2/11.3 ratios. Although the statistics are poor, small 6.2/11.3 ratios are found for most of the lowest-metallicity BCDs. Because the $11.3 \mu \mathrm{m}$ feature arises from non-adjacent or "solo" CH groups, dominant $11.3 \mu \mathrm{m}$ emission, like $8.6 \mu \mathrm{m}$ emission, implies large PAH species, with $\gtrsim 100-200$ carbon atoms and long straight edges (Hony et al. 2001; Bauschlicher et al. 2008; Bauschlicher et al. 2009). Moreover, fewer small PAHs reduce the intensity of the $6.2 \mu \mathrm{m}$ emission, as the bulk of this feature comes from PAHs containing less than $100 \mathrm{C}$ atoms (Schutte et al. 1993; Hudgins et al. 2005). Therefore, small $6.2 / 11.3 \mu \mathrm{m}$ band ratios at low metallicities could imply an overall deficit of small PAHs.

A third indication that there is a deficit of small PAHs in a metal-poor ISM is the intensity of the $8.6 \mu \mathrm{m}$ feature relative to the $7.7 \mu \mathrm{m}$ blend. As mentioned above, Bauschlicher et al. (2008) find that $8.6 \mu \mathrm{m}$ band arises from large PAHs, with $N_{\min } \gtrsim 100$ carbon atoms. On the other hand, the $7.7 \mu \mathrm{m}$ feature contains emission from both small and large PAH sizes (Schutte et al. 1993; van Diedenhoven et al. 2004). For the $9 \mathrm{BCDs}$ with the 7.7 and $8.6 \mu \mathrm{m} \mathrm{PAH}$ features detected at $\gtrsim 3 \sigma$, the mean $8.6 / 7.7$ flux ratio is 0.48 , and the median is 0.33 . This ratio is at least double that in the more metal-rich SINGS galaxies, with 8.6/7.7 0.18 in the mean, and a range of $0.11-0.21$ (Smith et al. 2007); 7 of 9 BCDs are outside the upper 90th percentile limit of 0.21 . Again, although the number statistics are small, the large 8.6/7.7 $\mu \mathrm{m}$ flux ratios of the PAHs in these low-metallicity BCDs appear to be dominated by the largest PAH species. 


\section{Conclusions}

In summary, there are three different lines of evidence which tentatively suggest that the PAH populations in low-metallicity BCDs are deficient in the smallest sizes $\left(N_{\min } \lesssim 50 \mathrm{C}\right.$ atoms $)$. The IRS spectra show flux ratios typical of the largest PAHs modeled so far, with $N_{\min } \gtrsim 100 \mathrm{C}$ atoms. Correlations within our sample suggest that the main trend is with radiation field intensity and hardness, rather than with metallicity. Apparently, the ISM environment in the BCD HiI regions is too harsh for the smallest PAHs, possibly destroying them through sputtering. Perhaps only the PAH population with the largest species can survive the extreme physical conditions in the low-metallicity HII regions in the BCDs.

We would like to thank the organizers for a very stimulating and well-organized conference.

\section{References}

Bauschlicher, C.W., Jr., Peeters, E., \& Allamandola, L.J., 2008, ApJ, 678, 316

Bauschlicher, C.W., Peeters, E., \& Allamandola, L.J., 2009, ApJ, 697, 311

Brandl, B. R., et al., 2006, ApJ, 653, 1129

Draine, B. T., \& Li, A., 2007, ApJ, 657, 810

Engelbracht, C.W., Rieke, G.H., Gordon, K.D., et al., 2008, ApJ, 678, 804

Galliano, F., Madden, S.C., Jones, A.P., Wilson, C.D., \& Bernard, J.-P., 2005, A\&A, 434,867

Hony, S., Van Kerckhoven, C., Peeters, E., et al., 2001, A\&A, 370, 1030

Houck, J.R., et al., 2004a, ApJS, 154, 18

Hudgins, D.M., Bauschlicher, C.W., Jr., \& Allamandola, L.J., 2005, ApJ, 632, 316

Hunt, L.K., Thuan, T.X., Izotov, Y.I., \& Sauvage, M., 2010, ApJ, 712, 164

Joblin, C., Boissel, P., Leger, A., D'Hendecourt, L., \& Defourneau, D., 1995, A\&A, 299, 835

Kennicutt, R.C., Jr., et al., 2003, PASP, 115, 928

Madden, S.C., Galliano, F., Jones, A.P., \& Sauvage, M., 2006, A\&A, 446, 877

Peeters, E., Hony, S., Van Kerckhoven, C., et al., 2002, A\&A, 390, 1089

Peeters, E., Spoon, H.W.W., \& Tielens, A.G.G.M., 2004a, ApJ, 613, 986

Sandstrom, K.M., Bolatto, A.D., Draine, B.T., Bot, C., \& Stanimirović, S., 2010, ApJ, 715,701

Schutte, W.A., Tielens, A.G.G.M., \& Allamandola, L.J., 1993, ApJ, 415, 397

Smith, J.D.T., et al., 2007, ApJ, 656, 770

Taylor, C.L., Kobulnicky, H.A., \& Skillman, E.D., 1998, AJ, 116, 2746

van Diedenhoven, B., Peeters, E., Van Kerckhoven, C., et al., 2004, ApJ, 611, 928

Verstraete, L., et al., 2001, A\&A, 372, 981

Wu, Y., Charmandaris, V., Hao, L., et al. 2006, ApJ, 639, 157 